\title{
Deprivation linked to malnutrition risk and mortality in hospital
}

\author{
Rebecca J. Stratton* and Marinos Elia \\ Institute of Human Nutrition, School of Medicine, University of Southampton, Southampton, UK
}

(Received 3 August 2005 - Revised 11 November 2005 - Accepted 20 April 2006)

\begin{abstract}
This study aimed to investigate the link between deprivation and in-hospital malnutrition and to assess any independent and interrelated effects of deprivation and malnutrition on clinical outcome in hospital. One thousand patients (mean age 71 (SD 19) years, mean BMI 25.6 (SD 5.4) kg/m²) were screened for malnutrition (using the Malnutrition Universal Screening Tool ('MUST')) and their clinical outcome assessed prospectively. The deprivation of patients' locality of residence prior to admission was recorded using the Index of Multiple Deprivation 2000 (IMD). Patients with medium and high malnutrition risk $(42 \%, n$ 420) were admitted from areas with significantly greater deprivation (lower ranks) than low-risk patients (IMD 3731 v. 3946; $P<0.02$ ). The prevalence of malnutrition increased by multiples of 1.14 (95\% CI 1.02, 1.28) for each increment in quartile of IMD rank. The odds of malnutrition of the most deprived quartile were greater than those of the least deprived quartile by a factor of 1.59 (95\% CI 1.11, 2.28). They were also greater for five of the six components of IMD deprivation (and by a factor of 1.73 (95\% CI 1.20, 2.49) for income and 1.69 (95\% CI 1.18, 2.42) for employment). Greater in-hospital mortality was associated with malnutrition, independently of IMD (or its individual components; odds ratio 2.04 (95\% CI 1.22, 3.44)). Length of stay was associated only with malnutrition risk $(P<0.0005)$. This study highlights that in-hospital malnutrition and deprivation are interrelated, yet have independent, adverse associations with patient outcome. Effective strategies are required to tackle these common health inequalities in both clinical and public health settings.
\end{abstract}

Malnutrition: Screening: Deprivation: Outcome Assessment

Reports have consistently highlighted the problems of health inequality, the adverse impact of poverty and deprivation on health, and the important role of nutrition (Department of Health and Social Security, 1980; Department of Health, 2001, 2004; Elia \& Stratton, 2005). Deprivation, including social, economic and environmental factors, may increase an individual's risk of developing disease-related malnutrition. This is a very common condition that has deleterious consequences on physical and psychological function and clinical outcome (Stratton et al. 2003b, 2006). Geographical inequality, including deprivation, has been shown to be associated with malnutrition and poorer nutrient status (Armstrong et al. 2005; Elia \& Stratton, 2005) and with poorer outcome (e.g. increased mortality) in the general population (Department of Health and Social Security, 1980; Acheson, 1998; Shaw et al. 2005). It is, however, unclear whether similar associations and interrelationships exist in the clinical setting between deprivation, malnutrition (which affects 15-60\% of hospital admissions; Stratton, 2005) and poor outcome in hospital (e.g. mortality, length of stay). This study therefore had two aims: to investigate the link between deprivation and malnutrition in a British hospital; and to assess any independent and interrelated effects of malnutrition and deprivation on clinical outcome in hospital.

\section{Subjects and methods}

Local Research Ethics Committee approval was obtained. Patients gave consent before being screened for malnutrition, and fewer than $2 \%$ of patients declined to participate during this 1-year study period. One thousand patients consecutively admitted to a university teaching hospital were included in the study from surgical ( $n$ 224), medical ( $n$ 255), elderly care ( $n$ 247) and trauma and orthopaedic (n 274) hospital wards (see Table 1 for details). Patients were screened for malnutrition risk using the Malnutrition Universal Screening Tool ('MUST'; Malnutrition Advisory Group, British Association for Parenteral and Enteral Nutrition; Elia, 2003; Fig. 1) within $48-72 \mathrm{~h}$ of admission to hospital as follows.

Height was measured to the nearest $0 \cdot 1 \mathrm{~cm}$ using a portable, free-standing stadiometer (SECA, Leicester, UK), according to standard methodology (Elia, 2003). If height could not be measured accurately (e.g. the person was unable to stand), recalled height (if reliable and realistic; Elia, 2003; Stratton et al. 2003a) or surrogate measures (e.g. knee height; Elia, 2000, 2003) were used to calculate height. Weight was measured to the nearest $0.01 \mathrm{~kg}$ using SECA clinical scales (conforming to EU Directive 90/384/ EEC). If weight could not be measured accurately, recalled weight (if reliable and realistic) was used (Elia, 2003; Stratton et al. 2003a). BMI $\left(\mathrm{kg} / \mathrm{m}^{2}\right)$ was calculated and scored accordingly (Fig. 1). If neither weight nor height could be obtained, subjective criteria assessing physical appearance (very thin, thin, etc.) were used. Percentage unplanned weight loss over 3-6 months was calculated, either from documented weights in patients' notes or from patients' reports, and were scored accordingly (Fig. 1). Subjective 
Table 1. Patient characteristics (n 1000)

\begin{tabular}{|c|c|c|}
\hline Characteristic & \multicolumn{2}{|c|}{ Value } \\
\hline \multirow{5}{*}{$\begin{array}{l}\text { Age } \\
\text { No. male } v \text {. female } \\
\text { BMI } \\
\text { Malnutrition risk (low, medium, high) } \\
\text { Index of Multiple Deprivation rank } \ddagger\end{array}$} & \multirow{4}{*}{\multicolumn{2}{|c|}{$\begin{array}{l}\text { Mean } 71(\text { SD } 19) \text { years* } \\
574 \text { v. } 426(57.4 \% \text { v. } 42 \cdot 6 \%) \dagger \\
\text { Mean } 25.6(\text { SD } 5 \cdot 4) \mathrm{kg} / \mathrm{m}^{2} \\
58 \%, 14 \%, 28 \%\end{array}$}} \\
\hline & & \\
\hline & & \\
\hline & & \\
\hline & Median & Range \\
\hline All patients ( $n$ 1000) & 3890 & $601-8375$ \\
\hline Surgical (n 224) & 3890 & $742-8375$ \\
\hline Medical ( $n$ 255) & 3731 & $603-8375$ \\
\hline Elderly care ( $n 247)$ & 3890 & $817-8375$ \\
\hline Trauma and orthopaedic ( $n$ 274) & 4100 & $601-8375$ \\
\hline
\end{tabular}

* Patients with a medium + high risk of malnutrition were older than those with a low risk (73 v. 69 years, $P<0.0005)$. There was no significant difference in age according to deprivation quartile.

†There was no significant differences in the ratio of men to women according to malnutrition risk or deprivation quartile.

‡Ranks range from 1 (greatest deprivation) to 8414 (least deprivation). For comparison, median IMD ranks are 4207 (nationally), 4404 (regionally; Southampton and South West Hampshire) and 6094 (locally; Southampton; Department of Environment, Transport and the Regions, 2000).

criteria could be used if reliable records or reports could not be obtained (Elia, 2003). An acute disease effect (if there had been or was to likely to have been no nutritional intake for over $5 \mathrm{~d}$ ) was noted and scored (Fig. 1).
The use of recalled and other alternative measures and subjective criteria as part of 'MUST' allowed all patients to be screened. 'MUST' has been validated with the use of these alternative and subjective measures, and previous work shows that the categorisation of malnutrition risk can be established with a sensitivity and specificity of $95 \%$ or more compared with the use of measured weight or height (Elia, 2003). For more information on the evidence base and use of 'MUST' and its practicality, reliability and concurrent and predictive validity, see Elia (2003) and Stratton et al. (2004, 2006).

A record was made of the prevalence of malnutrition risk using 'MUST' (low, medium and high risk, combined into two risk categories: low, and medium + high risk), and clinical outcome (mortality, length of hospital stay) was prospectively recorded. At the time of the study, nutritional screening was not routinely carried out on these hospital wards. The results of screening in the present study were not divulged to the nursing staff so that care continued routinely according to local policy.

Deprivation (for this study defined as 'an unmet need caused by a lack of resources of all kinds, including financial resources'; Office of the Deputy Prime Minister, 2004) was assessed using the Index of Multiple Deprivation (IMD) 2000, produced by the Department of Environment, Transport and the Regions (2000). This index comprehensively assesses

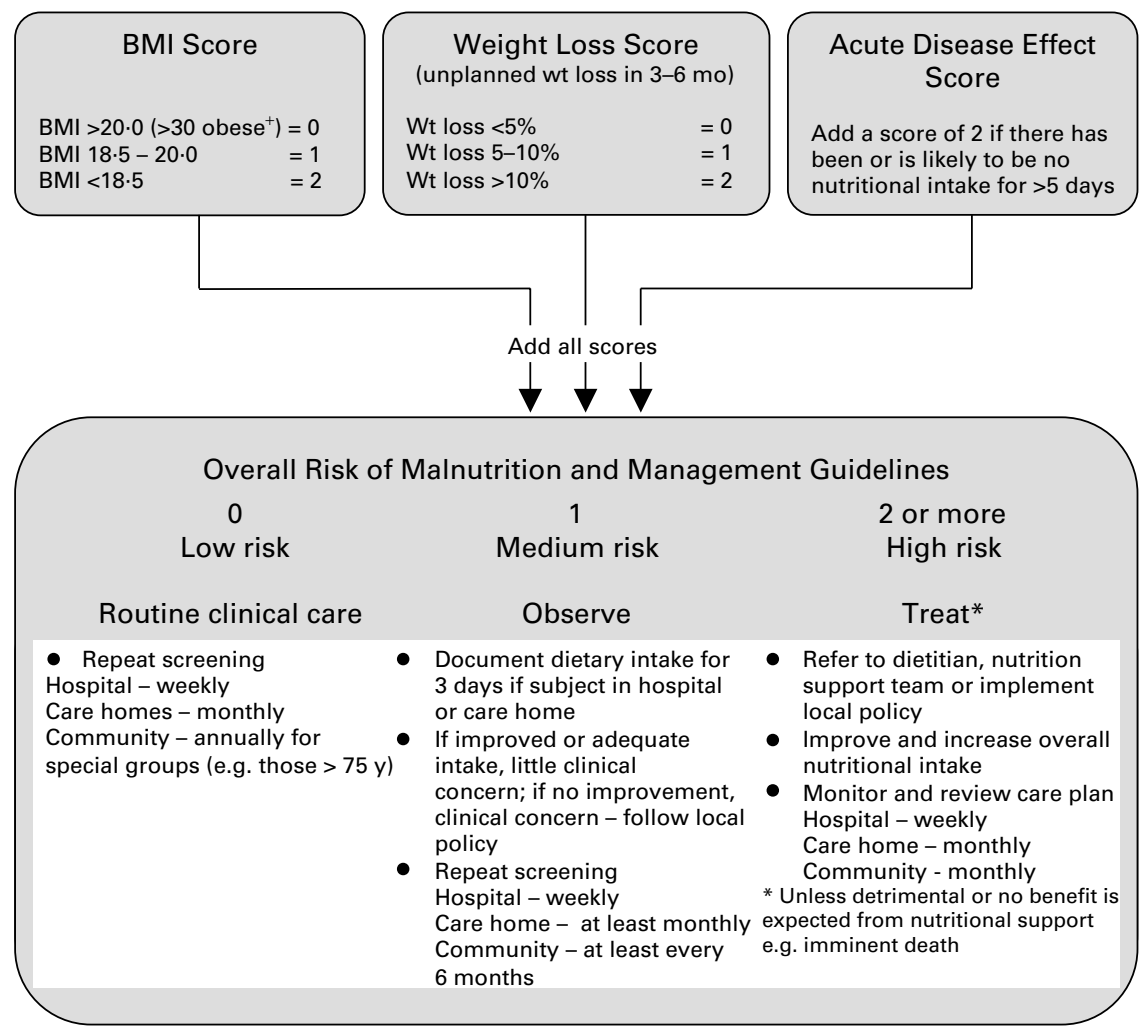

Record malnutrition risk category, presence of obesity and/or need for special diets and follow local policy. Reassess those identified at risk as they move through care settings ${ }^{+}$In the obese, underlying acute conditions are generally controlled before the treatment of obesity.

If unable to obtain height and weight, alternative measurements and subjective criteria are provided (Elia, 2003). (c) BAPEN 2003

Fig. 1. The Malnutrition Universal Screening Tool ('MUST'). 
six components (domains) of deprivation related to: income; employment; health deprivation and disability; education, skills and training; housing; access to services. These domains are then weighted (Table 2) and combined to form the overall index of multiple deprivation (IMD). For more information on the IMD domains and how they are derived, see Table 2.

The IMD is not specific to individuals, but to geographical areas (wards). There are 8414 geographical wards in England, which are ranked in order of deprivation across the whole country. The most deprived ward for each of the domains is given a rank of 1 and the least deprived a rank of 8414 . In the present study, the postcode of the residing address of all screened patients was used to determine the geographical ward they were admitted from and its associated deprivation. Table 1 shows the median IMD rank for the whole patient group ( $n$ 1000) and according to hospital ward type. As there were no significant differences between hospital ward types (surgical, medical, elderly, trauma and orthopaedics), these were combined and analysed as one group.

\section{Statistical analysis}

Indices of deprivation were analysed as ranks and as quartiles from the least deprived (most affluent; quartile 1) through to the most deprived (quartile 4; Table 3). They were also ranked according to national quartiles. Kruskal-Wallis one-way ANOVA and Mann-Whitney U non-parametric tests were undertaken to assess the differences in deprivation ranks between groups (i.e. according to malnutrition risk category or hospital speciality). Length of stay, adjusted for mortality, was assessed using Kaplan-Meier survival analysis (log rank test) or Cox regression analysis (to assess covariates such as age, sex, etc.). Differences in the proportions (i.e. the proportion of patients with malnutrition) were analysed using the chi-squared test. The influence of multiple variables (including age and sex) on binary outcomes (malnutrition $v$. no malnutrition; dead $v$. not dead) was assessed using binary logistic regression. Other potential confounders, such as smoking or severity of illness, were not recorded in this study so could not be assessed.

To examine the null hypothesis that the exposure effect for the ordered categorical variables (quartiles of deprivation) was linear, a log likelihood ratio statistic was used, which was based on two types of binary logistic regression analysis: assuming linearity, and allowing the exposure effect (the quartiles) to be non-linear (which produces an increase in $2 \mathrm{df}$ ); (Kirkwood \& Sterne, 2005). A value of $P<0.05$ was considered to be significant. Data are presented as means and standard deviations, or medians and range. Analysis was undertaken using SPSS statistical software package version 11.0 (SPSS, Woking, Surrey, UK).

\section{Results}

\section{Link between deprivation and malnutrition in hospital}

Patients at risk of malnutrition (42\%; $n$ 420/1000); $14 \%$ medium risk, $28 \%$ high risk) were admitted from areas of greater deprivation than those not at risk. The IMD ranks were significantly lower (indicating more deprivation; 3731 (2251-6826)) for those at risk of malnutrition with 'MUST' (medium + high risk) than those not at risk (low risk, 3946
(603-8375)) on admission to hospital (Fig. 2). Each of the individual 'MUST' components showed a similar, significant relationship with IMD (BMI $(P<0.03)$, percentage weight loss in 3-6 months $(P<0 \cdot 05)$, acute disease effect $(P<0 \cdot 05)$; Fig. 2$)$.

The prevalence of malnutrition risk (medium + high risk) significantly increased with each quartile of deprivation rank (IMD; predicted odds ratio 1.14 (95\% CI 1.02, 1.28), binary logistic regression model (low $v$. medium + high risk), adjusted for age and sex; Table 4). The odds ratio for the IMD (odds of the most deprived quartile (49\% malnourished)/odds of the least deprived quartile (38\% malnourished)), when adjusted for age and sex using binary logistic regression, was found to be 1.59 (95\% CI 1.11, 2.28; Table 3). The odds ratios according to quartile of deprivation rank (IMD and individual domains) are shown in Table 4. The table also shows results assuming that the ordered categorical exposure effects (the quartiles of deprivation) are linear (see the table footnote for tests of linearity). Of the individual domains, health deprivation and disability, income and employment deprivation were also significantly greater with increased malnutrition risk (Table 4). Analysis of data according to national quartiles of the IMD and its components gave results similar to those based on local deprivation quartiles. For example, for malnutrition the odds ratio (most to least deprived national quartiles) was 1.56 (95\% CI 1.09, 2.24) instead of 1.59 (95\% CI 1.11, 2.28; Table 4).

\section{Effects of deprivation and malnutrition on outcome in hospital}

Mortality. Patients who died during hospitalisation tended to live in more deprived areas (4210 adjusted for age and sex) than those who were alive on discharge (4210 v. 3674; $P=0 \cdot 06)$. In addition, the binary logistic model tended to show greater mortality among those living in more deprived areas (Table 5). When malnutrition risk was included as a covariate in the binary logistic regression model, the odds ratios (with the least deprived quartile as the reference quartile) remained virtually identical to those shown in Table 5. Malnutrition was also found to be related to mortality (odds ratio $2.59 ; 95 \%$ CI 1.59, 4.24; unadjusted) even when adjustments were made for age and sex (odds ratio 2.03; $95 \%$ CI 1.22, 3.39) or age, sex and IMD quartiles (odds ratio 2.07; $95 \%$ CI 1.03, 4.14), or age, sex and individual components of IMD deprivation (the odds ratio remaining virtually identical to that observed with IMD deprivation quartiles).

Analysis of data according to national deprivation quartiles produced broadly similar results to those based on local deprivation quartiles, which are shown in Tables 4 and 5. For example, for mortality (adjusted for age and sex), the odds ratio using national quartiles (most deprived to least deprived quartile) was found to be 1.63 (95\% CI $0.78,3.39)$ instead of 1.69 (95\% CI 0.81, 3.52; Table 5).

Length of stay. There was no significant difference in length of hospital stay between deprivation quartiles (e.g. 22 $(17-27) \mathrm{d} v .23$ (19-26) d for most $v$. least deprived quartile, adjusted for mortality) in a Kaplan-Meier survival analysis (log rank test; $P>0.05$ ) or in a Cox regression model (adjusted for age and sex, \pm malnutrition risk; $\mathrm{P}>0.05)$. In contrast, there was a significant difference in length of stay between malnutrition risk categories (e.g. low risk 10 (9-11) d, medium + high risk $15(13-17)$ d) in a Kaplan-Meier 
Table 2. Indicators and domains of deprivation in the Index of Multiple Deprivation (IMD) 2000

\begin{tabular}{|c|c|}
\hline Domain & Indicators \\
\hline \multirow{7}{*}{$\begin{array}{l}\text { Income - measures people who are on a low income } \\
\qquad(25 \% \text { weighting in IMD) }\end{array}$} & Adults in Income Support households (1998) \\
\hline & Children in Income Support households (1998) \\
\hline & Children in Income-based Job-seekers Allowance households (1998) \\
\hline & Adults in Family Credit households (1999) \\
\hline & Children in Family Credit households (1999) \\
\hline & Adults in Disability Working Allowance households (1999) \\
\hline & $\begin{array}{l}\text { Non-earning, non-Income Support pensioners and disabled Council } \\
\text { Tax Benefit recipients (1998) }\end{array}$ \\
\hline \multirow{5}{*}{$\begin{array}{l}\text { Employment - measures forced exclusion from work (people who want } \\
\text { to work but are unable to do so through unemployment, sickness or } \\
\text { disability) ( } 25 \% \text { weighting in IMD) }\end{array}$} & $\begin{array}{l}\text { Unemployment claimant counts (average of May 1998, August 1998, } \\
\text { November 1998, February 1990) }\end{array}$ \\
\hline & People out of work but in TEC-delivered, government-supported training \\
\hline & People aged $18-24$ on New Deal options \\
\hline & Incapacity Benefit recipients aged $16-59$ years $(1998)$ \\
\hline & Severe Disablement Allowance claimants aged $16-59$ years (1999) \\
\hline (15\% weighting in IMD) & People receiving Attendance Allowance or Disability Living Allowance (1998) \\
\hline & $\begin{array}{l}\text { Proportion of people of working age (16-59 years) receiving Incapacity } \\
\text { Benefit of Severe Disablement Allowance }(1998-9)\end{array}$ \\
\hline & Age- and sex-standardised ratio of limiting long-term illness (1991) \\
\hline & Proportion of births of low birth weight $(<2500 \mathrm{~g})(1993-7)$ \\
\hline \multirow{6}{*}{$\begin{array}{l}\text { Education, skills and training - measures education deprivation } \\
\qquad(15 \% \text { weighting in IMD) }\end{array}$} & Working-age adults with no qualifications (1995-8) \\
\hline & Children aged 16 and over not in full-time education (1999) \\
\hline & $\begin{array}{l}\text { Proportions of the } 17-19 \text {-year-old population who have not successfully } \\
\text { applied for higher education (1997-1998) }\end{array}$ \\
\hline & Key stage 2 primary school performance data (1998) \\
\hline & Primary-school children with English as an additional language (1998) \\
\hline & Absenteeism at primary level (all absences) (1998) \\
\hline \multirow{3}{*}{$\begin{array}{l}\text { Housing - identifies people living in unsatisfactory housing, and } \\
\text { homelessness ( } 10 \% \text { weighting in IMD) }\end{array}$} & Homeless households in temporary accommodation (1997-8) \\
\hline & Household overcrowding (1991) \\
\hline & Poor private sector housing (1996) \\
\hline \multirow{2}{*}{$\begin{array}{l}\text { Geographical access to services - measures access } \\
\text { to essential services ( } 10 \% \text { weighting in IMD) }\end{array}$} & Access to a post office (1998) \\
\hline & Access to food shops (1998) \\
\hline
\end{tabular}

For more information, see Department of Environment, Transport and the Regions (2000).

survival analysis (log rank test; $P<0.0005)$ and in a Cox regression model $(P<0.0005$ adjusting for mortality, age and sex, \pm quartile of deprivation rank on the IMD).

\section{Discussion}

This study of health inequality is the first to indicate a significant relationship between malnutrition in a British hospital (identified with 'MUST') and deprivation (using IMD (2000)) and to show significant but independent, adverse associations of malnutrition and deprivation with clinical outcome (mortality).

This study in a mixed cohort of hospital admissions (medical, surgical, elderly care, trauma and orthopaedic), highlights a new and interesting relationship between malnutrition in a British hospital and deprivation (Table 4, Fig. 2). Specifically, individuals who are malnourished (medium + high risk) on admission to hospital are admitted from areas of significantly greater deprivation than those at low risk. Both malnutrition risk categorisation ('MUST') and the individual components of 'MUST' are significantly related to deprivation (IMD; Fig. 2).

Deprivation is multifaceted, and the IMD2000 assesses a number of the components of deprivation (Table 2). In this study, prevalence of malnutrition increased significantly with the index of multiple deprivation (IMD) and several of its domains, such as health and disability, income and employment (Table 4). One of these, poor health and disability, a domain that represents long-standing illness (Table 2), could adversely impact on nutritional status by direct effects, as well as by indirect effects mediated through reduced appetite and the ability to purchase, prepare and eat food. All of the individuals in this study were acutely or chronically ill requiring hospitalisation, although disease severity was not assessed. Deprivation in income and employment were also increased in the malnourished, raising issues about food insecurity. Food insecurity exists when there is uncertainty that food will be available, or when there is an inability to access the available food because of financial or physical limitations (Bukhari et al. 2004). In our group of patients, however, it is unclear to what extent food insecurity existed as part of deprivation or whether it contributed to malnutrition. Other domains of deprivation assessed in the IMD, such as poor housing and education, may also negatively impact dietary intake (variety, nutritional adequacy), by adversely affecting the ability to choose, purchase and prepare a nutritionally 
Table 3. Summary of quartiles of deprivation

(Median and range)

\begin{tabular}{|c|c|c|c|c|c|c|c|c|c|c|}
\hline \multirow[b]{2}{*}{ Deprivation index } & \multicolumn{2}{|c|}{ All } & \multicolumn{2}{|c|}{$\begin{array}{c}\text { 1st quartile } \\
\text { (Least deprived) }\end{array}$} & \multicolumn{2}{|c|}{ 2nd quartile } & \multicolumn{2}{|c|}{ 3rd quartile } & \multicolumn{2}{|c|}{$\begin{array}{c}\text { 4th quartile } \\
\text { (Most deprived) }\end{array}$} \\
\hline & Median & Range & Median & Range & Median & Range & Median & Range & Median & Range \\
\hline $\begin{array}{l}\text { Index of multiple } \\
\text { deprivation* }\end{array}$ & 3890 & $601-8375$ & 7319 & $6355-8375$ & 5290 & $3946-6326$ & 2688 & $2487-3890$ & 1282 & $601-2251$ \\
\hline Housing & 2544 & $366-8337$ & 7100 & $5419-8337$ & 3943 & $2544-5398$ & 1589 & $1085-2498$ & 767 & $366-1012$ \\
\hline $\begin{array}{l}\text { Health deprivation } \\
\text { and disability }\end{array}$ & 4029 & $928-8396$ & 7243 & $6251-8396$ & 5370 & $4516-6133$ & 3368 & $2956-4476$ & 2156 & $928-2951$ \\
\hline Income & 3237 & $413-8401$ & 7021 & $6241-8401$ & 4265 & $3237-6236$ & 2578 & $2265-3118$ & 1502 & $413-2185$ \\
\hline Employment & 3946 & $361-8401$ & 7172 & $6015-8401$ & 4932 & $4082-5944$ & 3530 & $2960-4043$ & 2076 & $361-2868$ \\
\hline $\begin{array}{l}\text { Education, skills } \\
\text { and training }\end{array}$ & 3607 & $88-8262$ & 6672 & $5693-8262$ & 4518 & $3607-5664$ & 2288 & $1242-3526$ & 756 & $88-1145$ \\
\hline $\begin{array}{l}\text { Geographical access } \\
\text { to services }\end{array}$ & 5501 & $190-8330$ & 7271 & $6409-8330$ & 5969 & $5501-6298$ & 4528 & $3454-5441$ & 2705 & $190-3423$ \\
\hline
\end{tabular}

There were no significant differences in age or sex across the deprivation quartiles.

${ }^{*}$ For comparison, median Index of Multiple Deprivation ranks are 4207 nationally, 4404 regionally; Southampton and South West Hampshire and 6094 locally; Southampton; Department of Environment, Transport and the Regions, 2000.

adequate diet, all of which can increase the risk of malnutrition developing.

Previous observations suggest that malnutrition (using 'MUST') and a range of nutrient deficiencies are linked to deprivation as well as to geographical regions across England (southern, central and northern parts of England; Elia \& Stratton, 2005). The present study suggests that inequalities also exist within a smaller geographical area in one region of England.

In contrast to all the other domains of deprivation, which were associated with risk of malnutrition, geographical access to services was 'better' in the malnourished hospital patients. The reasons for this are unclear, although it could be that some of the services assessed (e.g. access to a primary school; Table 2) may not be relevant or that investment into improving inequalities in access to services had already begun. In addition, a recent study has highlighted the limited predictive power of this domain, especially in rural areas (Niggebrugge et al. 2005).

This study highlights the fact that disease-related malnutrition and deprivation are independently related to in-hospital mortality (Table 5), although only malnutrition is significantly associated with increased length of hospital stay. Many studies have indicated the relationship between disease-related malnutrition and increased risk of mortality and longer hospital stay (Stratton et al. 2006), and in the present study, greater mortality (by 6\%) and longer hospital stays (by $5 \mathrm{~d}$ ) were found to occur in patients with malnutrition. Systematic reviews and meta-analyses have also shown that nutritional interventions, such as multinutrient supplements that treat malnutrition, reduce mortality (see Stratton et al. 2003b; for a review). Similarly, individuals living in more deprived areas have been shown to have poorer life expectancy and greater morbidity (Department of Health and Social Security, 1980; Acheson, 1998; Shaw et al. 2005), so it is unsurprising that such individuals also have a poorer outcome in hospital.

Although the effects of deprivation on patient outcome might be explained by the associated malnutrition, as discussed above, our analysis suggested that deprivation also had effects on outcome that were independent of malnutrition. In this study, however, only protein-energy malnutrition was assessed (using 'MUST'). It is possible that those from more deprived localities had other nutritional problems, such as deficiencies of vitamins or other micronutrients, that might have detrimentally influenced patient outcomes independently of protein-energy status. Certainly, older subjects in the general population, who live in more deprived geographical areas in England, are more likely to have nutrient deficiencies, which could in turn detrimentally affect outcome, independently of protein-energy status (Elia \& Stratton, 2005). Unfortunately, nutrient status was not measured in this study.

Deprivation may adversely affect clinical outcome in other ways (e.g. through lifestyle and dietary factors) that may predispose to the development of more severe disease and later presentation for diagnosis and treatment. Other studies have shown that those from more deprived areas are less likely to accept

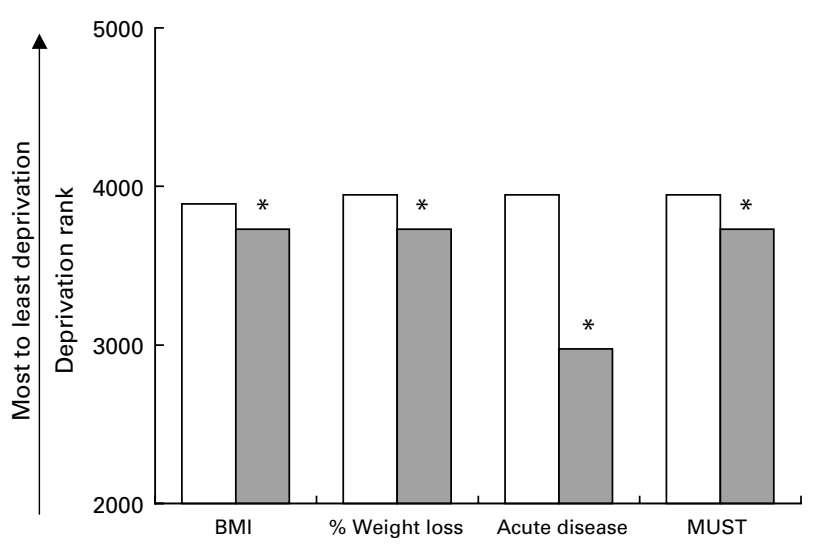

Fig. 2. Relationship between deprivation (Index of Multiple Deprivation 2000) and malnutrition risk (Malnutrition Universal Screening Tool ('MUST') and 'MUST' components) in patients admitted to hospital, lower ranks indicating greater deprivation*. There was significantly greater deprivation (lower ranks) for patients at risk of malnutrition ('MUST' and 'MUST' components; see Fig. 1; $P<0.05$ using the Mann-Whitney $U$ test). 'MUST' components: BMI score 0 $\left(\mathrm{BMl}>20 \mathrm{~kg} / \mathrm{m}^{2}\right) \mathrm{v}$. score 1 or more $\left(\mathrm{BMl}<20 \mathrm{~kg} / \mathrm{m}^{2}\right)$; percentage weight loss score 0 ( $<5 \%$ weight loss) $v$. score 1 or more ( $>5 \%$ weight loss); acute disease effect score 0 (none) $v$. score 2 (acute disease effect present); 'MUST' score 0 (low risk, $\square$ ) v. score 1 or more (medium + high risk, $\square$ ). 
Table 4. Malnutrition risk (medium + high $v$. low risk) by quartile of (local) deprivation rank (adjusted for age and sex)

\begin{tabular}{|c|c|c|c|c|c|c|c|c|c|}
\hline \multirow[b]{3}{*}{ Deprivation index } & \multicolumn{7}{|c|}{ Quartile* } & & \\
\hline & \multirow{2}{*}{$\begin{array}{c}\text { 1st } \\
\text { (Least deprived) }\end{array}$} & \multicolumn{2}{|c|}{$2 n d$} & \multicolumn{2}{|r|}{ 3rd } & \multicolumn{2}{|c|}{$\begin{array}{c}\text { 4th } \\
\text { (Most deprived) }\end{array}$} & \multicolumn{2}{|c|}{ Linear model† } \\
\hline & & OR & $95 \% \mathrm{Cl}$ & OR & $95 \% \mathrm{Cl}$ & OR & $95 \% \mathrm{Cl}$ & OR & $95 \% \mathrm{Cl}$ \\
\hline Index of multiple deprivation & $1 \cdot 00$ & $1 \cdot 01$ & $0.84,1.42$ & $1 \cdot 04$ & $0.73,1.50$ & 1.59 & $1 \cdot 11,2 \cdot 28$ & $1 \cdot 14$ & $1.02,1.28$ \\
\hline Housing & 1.00 & 0.93 & $0.65,1.33$ & 0.96 & $0.66,1.39$ & 1.36 & $0.95,1.94$ & $1 \cdot 10$ & $0.99,1.24$ \\
\hline Income & 1.00 & 1.41 & $0.99,2.01$ & 1.07 & $0.74,1.55$ & 1.73 & $1 \cdot 20,2 \cdot 49$ & $1 \cdot 15$ & $1.04,1.28$ \\
\hline Employment & 1.00 & $1 \cdot 25$ & $0.87,1.81$ & $1 \cdot 11$ & $0.77,1.61$ & 1.69 & $1 \cdot 18,2 \cdot 42$ & $1 \cdot 16$ & $1.03,1.30$ \\
\hline Education, skills and training & 1.00 & 0.81 & $0.56,1.16$ & 0.78 & $0.55,1.12$ & $1 \cdot 26$ & $0.88,1.82$ & - & - \\
\hline Geographical access to services & 1.00 & $1 \cdot 12$ & $0.79,1.60$ & 0.90 & $0.63,1.29$ & 0.81 & $0.57,1.15$ & 0.92 & $0.82,1.03$ \\
\hline
\end{tabular}

OR, odds ratio.

* The odds of malnutrition of each quartile ((medium + high risk)/low risk) is compared with that of the least deprived quartile (i.e. 1 st quartile $=$ reference quartile). Odds ratio $=$ odds of specified quartile/odds of 1 st quartile.

†The results shown represent the multiples by which the odds of malnutrition increases for each quartile increase in deprivation rank. (Odds ratio $=$ predicted odds of a quartile/predicted odds of the previous quartile using binary logistic modelling). Results are only shown when there is no statistical evidence against the null hypothesis that the quartile of deprivation is linear (in the binary logistic model $P>0.2$ for all indices of deprivation except for income, for which $P>0.05$ and $P<0.1$ ).

invitations for screening (e.g. for colorectal cancer; Whynes et al. 2003) and less likely to attend follow-up appointments (Johnson et al. 2004). Deprivation, including poorer education, may also negatively affect patients' responsiveness and compliance with prescribed treatments. Alternatively, individuals from deprived localities may be treated differently by hospital staff. We did not collect data to investigate these possibilities in our study. Other studies (in which nutrition was not considered) have linked deprivation to poorer hospital outcome and increased hospital admission rates (Hawker et al. 2003; Hutchings et al. 2004; Lyratzopoulos et al. 2005). The underlying mechanisms and the role of nutrition require investigation.

The lack of a demonstrable relationship between length of stay and deprivation may be due to an inadequate sample size ( $n$ 1000), especially since it is widely acknowledged that many factors, both clinical and social, affect the time of hospital discharge. For example, attitudes to recovery are important, but whether such attitudes are related to deprivation, independently of malnutrition risk, is uncertain.

The IMD was chosen as the overall deprivation index not only because it is widely recognised and used (e.g. Woods et al. 2005), but also because it has been shown to have good agreement with other commonly used tools, for example the Jarman Underprivileged Area Score (1991), developed to measure general practitioner workload (NHS Executive, 2000), and the Townsend Score (1988; Townsend et al. 1988; Jordan et al. 2004). It is easy to use and assesses a range of deprivation domains (Table 2). One of the limitations of the IMD(2000) is, however, that it identifies the deprivation of the locality (geographical ward) in which an individual lives (derived from postcode) rather than the deprivation of individuals per se. The newer indices of deprivation (published after the commencement of this study) assess deprivation in smaller geographical areas called super-output areas (within wards) and include additional domains (including crime). These should be used in the future to identify more closely the relationship between malnutrition and deprivation (Office of the Deputy Prime Minister, 2004).

Another limitation of this study is that the potential confounding effects of smoking and disease severity were not addressed. Furthermore, this investigation did not continue to monitor the healthcare use of this patient group after discharge; this is an

Table 5. Mortality (dead v. not dead) by quartile of (local) deprivation rank

\begin{tabular}{|c|c|c|c|c|c|c|c|c|c|}
\hline \multirow[b]{3}{*}{ Deprivation index } & \multicolumn{7}{|c|}{ Quartile* } & & \\
\hline & \multirow{2}{*}{$\begin{array}{c}\text { 1st } \\
\text { (Least deprived) }\end{array}$} & \multicolumn{2}{|c|}{ 2nd } & \multicolumn{2}{|c|}{ 3rd } & \multicolumn{2}{|c|}{$\begin{array}{c}\text { 4th } \\
\text { (Most deprived) }\end{array}$} & \multicolumn{2}{|c|}{ Linear model† } \\
\hline & & OR & $95 \% \mathrm{Cl}$ & OR & $95 \% \mathrm{Cl}$ & OR & $95 \% \mathrm{Cl}$ & OR & $95 \% \mathrm{Cl}$ \\
\hline Index of multiple deprivation & 1.00 & 0.71 & $0.31,1.63$ & $2 \cdot 07$ & $1.03,4.14$ & 1.69 & $0.81,3.52$ & $1 \cdot 31$ & $1.04,1.65$ \\
\hline Housing & 1.00 & 1.03 & $0.48,2.18$ & 1.50 & $0.73,3.07$ & $1 \cdot 77$ & $0.87,3.61$ & $1 \cdot 24$ & $0.99,1.55$ \\
\hline Income & 1.00 & 1.44 & $0.69,3.00$ & $1 \cdot 73$ & $0.83,3.63$ & 1.83 & $0.85,3.94$ & $1 \cdot 21$ & $0.96,1.53$ \\
\hline Employment & 1.00 & 1.79 & $0.80,4.01$ & $2 \cdot 29$ & $1.04,5.04$ & $2 \cdot 17$ & $0.98,4.83$ & $1 \cdot 26$ & $1.00,1.58$ \\
\hline Education, skills and training & 1.00 & 1.09 & $0.51,2.34$ & $1 \cdot 75$ & $0.88,3.49$ & $1 \cdot 74$ & $0.83,3.65$ & $1 \cdot 24$ & $0.99,1.56$ \\
\hline Geographical access to services & 1.00 & 0.71 & $0.38,1.33$ & 0.39 & $0.19,0.80$ & 0.37 & $0.18,0.77$ & 0.69 & $0.55,0.87$ \\
\hline
\end{tabular}

OR, odds ratio.

${ }^{*}$ The odds of malnutrition of each quartile $(($ medium + high risk $) /$ low risk) is compared with that of the least deprived quartile (i.e. 1 st quartile $=$ reference quartile). Odds ratio $=$ odds of specified quartile/odds of 1st quartile.

†The results shown represent the multiples by which the odds of malnutrition increases for each quartile increase in deprivation rank. (Odds ratio $=$ predicted odds of a quartile/predicted odds of the previous quartile using binary logistic modelling). There was no statistical evidence against the null hypothesis that quartile of deprivation was linear (in the binary logistic model $P>0.3$ for all indices of deprivation except for Index of Multiple Deprivation 2000, for which $P>0.05$ and $P<0.1$ ). 
area for further investigations, especially as an earlier study suggested a greater need for primary care in deprived areas (Carlisle et al. 2002). Finally, results obtained using a population served by only one hospital should only be extrapolated to the whole country with caution as patient history, standards of care, length of hospital stay and in-patient mortality vary from hospital to hospital. Nevertheless, when deprivation was categorised according to national rather than local quartiles of deprivation, broadly similar results were obtained.

Solutions to inequity problems are likely to be complex and may require changes not only in the healthcare sector, but also in social and economic factors that lie outside the healthcare sector. Public-health policies need to consider the graded effects of deprivation on outcomes. In this study, the increase in mortality or malnutrition with increasing quartiles of deprivation did not appear to be progressive for some domains. However, the overall pattern of increase did not differ significantly from linearity (binary logistic regression) except for the education domain, when binary logistic regression was used with malnutrition as the dependent variable. Among the factors that can influence the overall pattern is sample size.

In conclusion, this is the first study to show that malnutrition risk on admission to a British hospital (using 'MUST') and the individual components of 'MUST' malnutrition risk (BMI less than $20 \mathrm{~kg} / \mathrm{m}^{2}$, more than $5 \%$ unintentional weight loss in 3-6 months, acute disease effect) are associated with deprivation, which was assessed using the $\operatorname{IMD}(2000)$ for England. In addition, the findings suggest that malnutrition and deprivation are independently linked to greater mortality in hospital. The causes, consequences and methods for tackling malnutritionrelated inequalities require further investigation.

\section{Acknowledgements}

This work was supported by Southampton University Hospitals NHS Trust Strategic Research and Development Funds. With thanks to the Clinical Nutrition Research and Development Team.

\section{References}

Acheson D (1998) Independent Enquiry into Inequalities in Health. London: The Stationery Office.

Armstrong J, Dorosty AR \& Reilly JJ, Child Health Information Team \& Emmett, PM (2005) Coexistence of social inequalities in undernutrition and obesity in preschool children: population based cross sectional study. Arch Dis Child 88, 671-675.

Bukhari HM, Margetts BM \& Jackson A (2004) Food insecurity in the UK; determinants and consequences. Asia Pac J Clin Nutr 13, S167.

Carlisle R, Avery AJ \& Marsh P (2002) Primary care teams work harder in deprived areas. $J$ Publ Health Med 24, 43-48.

Department of Environment, Transport and the Regions (2000) Indices of Deprivation 2000. London: DETR.

Department of Health (2001) Tackling Health Inequalities. A Programme for Action. London: Department of Health.

Department of Health (2004) Choosing Health? Choosing a Better Diet. A Consultation on Priorities for a Food and Health Action Plan. London: NHS Estates, Department of Health.

Department of Health and Social Security (1980) Inequalities in Health: Report of a Research Working Group (Black Report). London: DHSS.
Elia M (ed.) (2000) Guidelines for Detection and Management of Malnutrition. Maidenhead: British Association for Parenteral and Enteral Nutrition, Malnutrition Advisory Group.

Elia M (2003) Screening for Malnutrition: A Multidisciplinary Responsibility. Development and Use of the Malnutrition Universal Screening Tool ('MUST') for Adults. Redditch: British Association for Parenteral and Enteral Nutrition.

Elia M \& Stratton RJ (2005) Geographical inequalities in nutrient status and risk of malnutrition among English people aged 65 years and over. Nutrition 21, 1100-1106.

Hawker JI, Olowokure B, Sufi F, Weinberg J, Gill N \& Wilson RC (2003) Social deprivation and hospital admission for respiratory infection: an ecological study. Respir Med 97, 1219-1224.

Hutchings A, Raine R, Brady A, Wildman M \& Rowan K (2004) Socioeconomic status and outcome from intensive care in England and Wales. Med Care 42, 943-951.

Johnson R, Horne B, Feltblower RG, Butler GE \& Glaser AW (2004) Hospital attendance patterns in long term survivors of cancer. Arch Dis Child 89, 374-377.

Jordan H, Roderick P \& Martin D (2004) The Index of Multiple Deprivation 2000 and accessibility effects on health. $J$ Epidemiol Commun Health 58, 250-257.

Kirkwood BR \& Sterne JAV (2005) Regression modelling. In Essential Medical Statistics, 2nd ed., pp. 315-342 Oxford: Blackwell Science.

Lyratzopoulos G, Havely D, Gemmell I \& Cook GA (2005) Factors influencing emergency medical readmission risk in a UK district general hospital: a prospective study. BMC Emerg Med 21, 1.

NHS Executive (2000) Deprivation in the South East Regional Office. An Analysis of the Department of the Environment, Transport and Regions Indices of Deprivation 2000. London: Department of Public Health Medicine.

Niggebrugge A, Haynes R, Jones A, Lovett A \& Harvey I (2005) The index of multiple deprivation 2000 access domain: a useful indicator for public health? Soc Sci Med 60, 2743-2753.

Office of the Deputy Prime Minister (2004) The English Indices of Deprivation 2004. London: Office of the Deputy Prime Minister.

Shaw M, Davey Smith G \& Dorling D (2005) Health inequalities and New Labour: how the promises compare with real progress. BMJ 330, 1016-1021.

Stratton RJ (2005) Elucidating effective ways to identify and treat malnutrition. Proc Nutr Soc 64, 305-311.

Stratton RJ, Dixon R, Longmore D, Stroud M \& Elia M (2003a) Effect of recalled weight and height on malnutrition risk. Clin Nutr 22, S9-S10.

Stratton RJ, Green CJ \& Elia M (2003b) Disease-related Malnutrition: An Evidence Based Approach to Treatment. Oxford: CABI Publishing.

Stratton RJ, Hackston A, Longmore D, Dixon R, Price S, Stroud M, King C \& Elia M (2004) Malnutrition in hospital outpatients and inpatients: prevalence, concurrent validity and ease of use of the 'Malnutrition Universal Screening Tool' ('MUST') for adults. $\mathrm{Br}$ J Nutr 92, 799-808.

Stratton RJ, King CL, Stroud MA, Jackson AA \& Elia M (2006) Malnutrition Universal Screening Tool ('MUST') predicts mortality and length of hospital stay in acutely ill elderly. Br J Nutr 95, 325-330.

Townsend P, Phillimore P \& Beattie A (1988) Health and Deprivation: Inequality and the Nort. London: Croom Helm.

Whynes DK, Frew EJ, Manghan CM, Scholefield JH \& Hardcastle JD (2003) Colorectal cancer, screening and survival: the influences of socio-economic deprivation. Publ Health 117, 289-295.

Woods LM, Rachet B, Riga M, Stone N, Shah A \& Coleman MP (2005) Geographical variation in life expectancy at birth in England and Wales is largely explained by deprivation. J Epidemiol Commun Health 59, 115-120. 